\title{
NUTRIÇÃO FOSFÁTICA E MICORRIZA ARBUSCULAR NA REDUÇÃO DA TOXICIDADE DE CÁDMIO EM TREMA [Trema micrantha (L.) Blum.] ${ }^{1}$
}

\author{
Cláudio Roberto Fonsêca Sousa Soares², José Oswaldo Siqueira ${ }^{3}$, Janice Guedes de Carvalho ${ }^{3}$, Luiz \\ Roberto Guimarães Guilherme ${ }^{3}$
}

\begin{abstract}
RESUMO - Neste trabalho, avaliaram-se os efeitos da nutrição fosfática e da micorriza arbuscular na toxicidade de Cd em Trema micrantha (L.) Blum. Em um primeiro experimento, mudas de trema foram formadas em substrato contendo doses crescentes de $\mathrm{P}\left(0,100,200\right.$ e $\left.400 \mathrm{mg} \mathrm{dm}^{-3}\right)$ e com um tratamento de inoculação com Glomus etunicatum. Após crescimento por 60 dias, essas mudas foram transferidas para solução nutritiva de Clark sem Cd e suplementada com 5, 15 e $45 \mu \mathrm{mol} \mathrm{L}^{-1}$ de Cd e mantidas por mais 40 dias, quando os efeitos dos tratamentos foram avaliados. As influências do P na amenização da fitotoxidez de $\mathrm{Cd}$ foram também avaliados em outro experimento, aplicando-se simultaneamente e de forma combinada em solução, doses de $\mathrm{P}(0,07$; 0,$5 ; 1 ;$ e $\left.2 \mathrm{mmol} \mathrm{L}^{-1}\right)$ e de $\mathrm{Cd}\left(0,5,10\right.$ e $\left.15 \mu \mathrm{mol} \mathrm{L}^{-1}\right)$. Houve acentuada inibição no crescimento e colonização

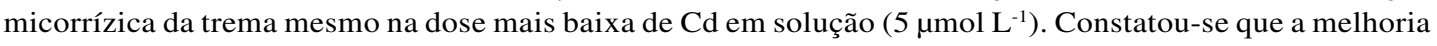
da nutrição fosfática favoreceu o crescimento da planta, sendo isso associado à redução da toxicidade de $\mathrm{Cd}$, enquanto a inoculação com G. etunicatum não teve o mesmo efeito. Encontrou-se uma relação inversa entre o aumento no fornecimento de $\mathrm{P}$ em solução e a absorção e acúmulo de $\mathrm{Cd}$ na trema. Análise da especiação química da solução indicou que o P não interferiu na disponibilidade de Cd em solução, o que evidencia que a ação amenizante do $\mathrm{P}$ se deve às interações na planta, possivelmente reduzindo a translocação do Cd.
\end{abstract}

Palavras-chave: Metais pesados, fosfato e fungo micorrízico.

\section{PHOSPHATE NUTRITION AND ARBUSCULAR MYCORRHIZA ON AMELIORATION OF CADMIUM TOXICITY IN TREMA [Trema micrantha (L.) Blum.].}

\begin{abstract}
In the present study the effects of phosphorus nutrition and arbuscular mycorrhiza (AM) were evaluated on Cd toxicity to Trema micrantha (L.) Blum.. In a first experiment, trema seedlings were raised in a P-enriched substrate $\left(0,100,200\right.$ and $\left.400 \mathrm{mg} \mathrm{dm}^{-3}\right)$ and with an AM treatment (inoculation with Glomus etunicatum). After sixty days of growth, plants were transferred to Clark nutrient solution either without Cd added or amended with this metal at concentration of 5, 15 and $45 \mu \mathrm{mol} \mathrm{L} \mathrm{L}^{-1}$. After the transplant to solution with treatments, plants were allowed to grow for additional forty days, when treatment effects were evaluated. Phosphorus effects on amelioration of Cd toxicity were also evaluated in a second experiment in which $P$ at concentration of 0.07; 0.5; 1 and $2 \mathrm{mmol} \mathrm{L}^{-1}$ and $C d$ at 0, 5, 10 and $15 \mu \mathrm{mol} \mathrm{L}^{-1}$ were combined and simultaneously applied in the growing nutrient solution. It was found that Cd exhibited high inhibition on growth and on AM colonization even at the lowest tested concentration ( $\left.5 \mu \mathrm{mol} L^{-1}\right)$. Enhanced P nutrition favored plant growth by reducing Cd concentration in plant shoots and consequently Cd phytotoxicity, whereas AM inoculation had no significant effect. An inverse relationship between P supply and Cd plant uptake and accumulation was found in the second experiment. The chemical speciation analysis of the nutrient solution showed that $P$ application had no significant interference on Cd available forms, therefore indicating that the $P$-ameliorating effect on Cd toxicity results from "in plant" interactions that cause reduction in translocation of Cd absorbed by the roots.
\end{abstract}

Keywords: Heavy metals, phosphate-metal interaction and mycorrhizal fungi.

\footnotetext{
${ }^{1}$ Recebido em 29.08.2006 e aceito para publicação em 29.03.2007. Parte da tese de Doutorado em Solos e Nutrição de Plantas do primeiro autor, apresentada à Universidade Federal de Lavras em novembro de 2004. Trabalho financiado pelo CNPq. ${ }^{2}$ Departamento de Ciência do Solo da Universidade Federal de Lavras (DCS/UFLA), Cx. Postal 37, 37200-000 Lavras-MG e Assistente de pesquisa PRODOC-Capes. E-mail: <crfsoares@gmail.com>.

${ }_{3}^{3}$ Departamento de Ciência do Solo da UFLA. Bolsistas do CNPq.E-mail: <siqueira@ufla.br>; <janicegc@ufla.br>; <guilherm@ufla.br>
} 


\section{INTRODUÇÃO}

A ciclagem biogeoquímica de metais pesados e o fluxo natural desses elementos na biosfera têm sido alterados em razão da crescente atividade antrópica nos ecossistemas (KABATA-PENDIAS e PENDIAS, 2001). Alguns metais pesados, como cádmio (Cd), chumbo $(\mathrm{Pb})$ e mercúrio $(\mathrm{Hg})$, não apresentam funções fisiológicas ou nutricionais nos seres vivos, mas são bem conhecidos como poluentes ambientais devido à possibilidade de bioacumulação na cadeia alimentar, causando danos a eles (CUI et al., 2005).

O Cd é universalmente conhecido pela sua elevada toxicidade para as plantas e animais (BARCELÓ e POSCHENRIEDER, 1992). Contaminações ambientais com Cd são freqüentes em áreas de processamento de rejeitos de minérios, composto de lixo e lodo de esgoto, indústrias de plástico e lubrificante e, em alguns casos, pela aplicação de fertilizantes fosfatados ricos em Cd (ALLOWAY, 1993; CAMPOS et al., 2005). No solo, a maior parte do Cd apresenta-se na forma trocável (RIBEIRO FILHO et al., 1999), sendo facilmente absorvido pelas raízes e causando sérios danos às plantas. A fitotoxicidade desse elemento em espécies arbóreas manifesta-se normalmente como cloroses foliares (SOARES et al., 2005) resultantes de efeitos fisiológicos, como diminuição da fotossíntese, inibição da respiração e transporte de elétrons nas mitocôndrias e diminuição da atividade enzimática (SANITÀ di TOPPI e GABBRIELLI, 1999). Entretanto, espécies vegetais e, mesmo, cultivares de uma mesma espécie diferem amplamente na habilidade de absorver, acumular, translocar e tolerar o excesso de Cd (YANG et al., 1995). Os mecanismos de tolerância das plantas ao Cd envolvem, principalmente, a produção de fitoquelatinas e a compartimentalização desse elemento no vacúolo (COBBETT, 2003), mas há especulações sobre o envolvimento de outros mecanismos que participam na proteção das plantas ao excesso desse metal como a colonização das raízes por fungos ectomicorrízicos (KRUPA e KOZDRÓJ, 2004) e micorrízicos arbusculares (MEHARG, 2003).

As micorrizas arbusculares (MA) podem contribuir para a tolerância das plantas ao excesso de metais pesados por meio de vários mecanismos físicos e biológicos (CHRISTIE et al., 2004). As estruturas fúngicas representam uma barreira biológica à toxicidade de metais, uma vez que estas podem imobilizar esses elementos (GAUR eADHOLEYA, 2004). Entretanto, as MAs podem ter efeitos variados na absorção de metais pesados pelas plantas, podendo aumentar a absorção ou inibila. Em estudo com ${ }^{109} \mathrm{Cd}$, Joner e Leyval (1997) verificaram que plantas de Trifolium subterraneum inoculadas com Glomus mosseae apresentaram menor translocação de Cd em comparação com aquelas não-inoculadas, indicando ser este um mecanismo importante de proteção das plantas. Além dos efeitos na absorção e translocação de $\mathrm{Cd}$, tem sido proposto que os fungos MAs podem contribuir indiretamente para a proteção das plantas contra a toxicidade de $\mathrm{Cd}$, aumentando a absorção de $\mathrm{P}$ que, normalmente, ocorre em resposta à micorrização, contribuindo, assim, para o crescimento das plantas sob estresse do metal (GAUR e ADHOLEYA, 2004). Isso, entretanto, ainda não foi investigado experimentalmente, sendo objeto deste estudo, que avaliou a influência da micorrização, do "status" nutricional de $\mathrm{P}$ e de concentrações crescentes de $\mathrm{P}$ em solução na toxicidade de Cd para mudas de Trema micrantha, uma espécie arbórea pioneira nos trópicos.

\section{MATERIAL E MÉTODOS}

Este estudo constou de dois experimentos distintos conduzidos com mudas de Trema micrantha (L.) Blum., desenvolvidos no Laboratório de Microbiologia do Solo da Universidade Federal de Lavras, no período de abril de 2002 a julho de 2003. Em um primeiro experimento, avaliaram-se os efeitos de micorriza arbuscular (MA) e do "status" nutricional de P na toxicidade de Cd. Para isso, mudas de trema foram cultivadas em substrato contendo tratamentos distintos para a obtenção de plantas com diferentes "status" nutricional de $\mathrm{P}$ e colonização com fungos micorrízicos arbusculares (micorriza). As mudas foram posteriormente transferidas para a solução nutritiva contendo doses crescentes de $\mathrm{Cd}$, estabelecidas com base em outros estudos desse laboratório (PAIVA et al., 2000). O experimento foi conduzido em casa de vegetação, utilizando-se mudas de trema, a qual foi selecionada por ser uma espécie pioneira de grande importância em programas de revegetação de áreas degradadas nos trópicos, por apresentar alta sensibilidade ao excesso de metais pesados (MARQUES et al., 2000) e também pelo fato de responder positivamente à inoculação com fungos micorrízicos e a doses de P (PARON et al., 1997). Para a obtenção das mudas, as sementes foram tratadas com $\mathrm{H}_{2} \mathrm{SO}_{4}$ concentrado durante 20 minutos e, em seguida, lavadas em água corrente, de modo a facilitar a germinação. Em seguida, foram colocadas em bandejas 
com capacidade de $6 \mathrm{dm}^{3}$ de um substrato autoclavado (40\% de um Latossolo Vermelho-Amarelo, $40 \%$ de areia e $20 \%$ de vermiculita), que apresentou as seguintes características: $\mathrm{pH}_{\text {(água) }}=6,1 ; \mathrm{P}=12,8 \mathrm{mg} \mathrm{dm}^{-3} ; \mathrm{K}=72$ $\mathrm{mg} \mathrm{dm}^{-3}$; e V (saturação por bases $)=70 \%$.

$\mathrm{O}$ substrato foi adubado com $\left[\mathrm{Ca}\left(\mathrm{H}_{2} \mathrm{PO}_{4}\right)_{2} \cdot \mathrm{H}_{2} \mathrm{O}\right.$ p.a. $]$ por meio da adição de solução nutritiva de modo a fornecer: 0, 100, 200 e $400 \mathrm{mg} \mathrm{dm}^{-3}$ de P, constituindo-se nos tratamentos prévios de P. Além desses, obteve-se um tratamento de inoculação com a aplicação de suspensão de esporos de Glomus etunicatum, de modo a se obter uma densidade de três esporos $\mathrm{mL}^{-1}$ de substrato. Os esporos foram extraídos de vasos de cultivo com Brachiaria decumbens, conforme metodologia sugerida por Gerdemann e Nicolson (1963). Em cada bandeja foram colocadas cerca de 500 sementes, as quais foram mantidas por 60 dias nos tratamentos (P e MA). Após esse período, selecionaram-se 10 plantas de cada bandeja para análise dos teores de nutrientes, de modo a verificar os efeitos da aplicação dos tratamentos, obtendo-se mudas com teores foliares médios de 2,1 a 4,2 $\mathrm{g} \mathrm{kg}^{-}$ ${ }^{1} \mathrm{de}$ P. Também foram coletadas amostras de raízes frescas (2g) para avaliação da colonização micorrízica das mudas. Para isso, as raízes foram tratadas conforme a metodologia proposta por Koske e Gemma (1989) e avaliadas pelo método das intersecções em placas reticuladas (GIOVANNETTI e MOSSE, 1980). Nessa etapa do estudo, as mudas apresentaram colonização média de $48 \% \pm$ 9\%. Em uma segunda etapa, mudas selecionadas e padronizadas foram transplantadas para bandejas com capacidade para $35 \mathrm{~L}$ contendo solução nutritiva de Clark (1975), preparada com reagentes PA e que continha: $7,26 \mathrm{mmol} \mathrm{L}^{-1} \mathrm{~N}-\mathrm{NO}_{3}^{-} ; 0,90 \mathrm{mmol} \mathrm{L}^{-1} \mathrm{~N}-\mathrm{NH}_{4}^{+} ; 0,07$ mmol L ${ }^{-1} \mathrm{P} ; 1,80 \mathrm{mmol} \mathrm{L}^{-1} \mathrm{~K} ; 2,60 \mathrm{mmol} \mathrm{L}^{-1} \mathrm{Ca} ; 0,60$ mmol L-1 Mg; 0,50 mmol L-1 S; 7,0 mmol L-1 $\mathrm{Mn} ; 2$ mmol L-1 $\mathrm{Zn} ; 0,5 \mathrm{mmol} \mathrm{L}^{-1} \mathrm{Cu} ; 19 \mathrm{mmol} \mathrm{L}^{-1} \mathrm{~B} ; 0,60 \mathrm{mmol}$ $\mathrm{L}^{-1}$ Mo e $38 \mathrm{mmol} \mathrm{L}^{-1} \mathrm{Fe}$ complexado com $\mathrm{Na}_{2}$-EDTA. Nessa etapa, a concentração de todos os nutrientes foi reduzida à metade ( $1 / 2$ força), a aeração foi constante e pH ajustado em 5,5 (adição de $\mathrm{NaOH}$ ou $\mathrm{HCl}$ 0,1 mol L ${ }^{-1}$ ), sendo nessas condições as plantas mantidas por quatro dias. Após nova padronização quanto à altura e ao vigor, transplantou-se uma muda por vaso de 1,7 L contendo a solução nutritiva de Clark com a concentração normal dos nutrientes e as doses de Cd $\left(0,5,15\right.$ e $45 \mu \mathrm{mol} \mathrm{L} \mathrm{L}^{-1} \mathrm{Cd}$, fornecido como $\mathrm{CdSO}_{4} .8 \mathrm{H}_{2} \mathrm{O}$ ). Os tratamentos foram dispostos em esquema fatorial $5 \times 4$ : cinco tratamentos prévios (doses de $\mathrm{P}$ e inoculação com G. etunicatum) e as quatro concentrações de Cd em blocos casualizados (DBC) com três repetições. As soluções foram renovadas semanalmente e o pH ajustado em 5,5 pela adição de $\mathrm{NaOH}$ ou $\mathrm{HCl} 0,1 \mathrm{~mol} \mathrm{~L}^{-1}$, sendo o experimento conduzido por um período de 40 dias.

No segundo experimento, avaliaram-se os efeitos de doses de $\mathrm{P}$ em solução na toxicidade de $\mathrm{Cd}$ para a trema. $\mathrm{O}$ ensaio foi também conduzido em casa de vegetação, porém consistindo-se na aplicação simultânea de doses de $\mathrm{Pe}$ de $\mathrm{Cd}$. As mudas de trema foram preparadas conforme descrito no primeiro experimento. Aos 60 dias após a germinação, as mudas foram transplantadas para bandejas coletivas de $35 \mathrm{~L}$ contendo a solução nutritiva de Clark ( $1 / 2$ força) e permanecendo nessa situação por 20 dias para aclimatação, quando atingiram altura média de $30 \mathrm{~cm}$, tamanho ideal para o transplante para o campo. Após esse período, as mudas foram selecionadas (altura e vigor) e transplantadas (uma muda por vaso de 1,7 L) contendo a solução nutritiva de Clark, ajustando-a de modo a obter diferentes concentrações de $\mathrm{P}\left(0,07 ; 0,5 ; 1 ;\right.$ e $\left.2 \mathrm{mmol} \mathrm{L}^{-1}\right)$ e de $\mathrm{Cd}(0,5,10$ e $15 \mu \mathrm{mol} \mathrm{L}-1)$ aplicadas simultaneamente e combinadas em esquema fatorial $4 \times 4 \mathrm{em} \mathrm{DBC}$, com três repetições. As doses de $\mathrm{P}$ foram definidas com base nas concentrações desse nutriente em solução nutritiva, sendo a concentração original da solução de Clark de 0,07 $\mathrm{mmol} \mathrm{L}^{-1} \mathrm{P}$. Enquanto isso, as doses de $\mathrm{Cd}$ foram definidas conforme concentrações empregadas em outros experimentos com espécies arbóreas (PAIVA et al., 2000). As soluções de todos os tratamentos foram renovadas semanalmente, o pH ajustado em 5,5 pela adição de $\mathrm{NaOH}$ ou $\mathrm{HCl} 0,1 \mathrm{~mol}$ $\mathrm{L}^{-1} \mathrm{e}$ as plantas conduzidas por 40 dias. Neste experimento, realizou-se também uma análise da especiação iônica da solução nutritiva de Clark contendo os diferentes tratamentos, empregando-se o software Visual Minteq v. 2.23. Para isso, foram utilizadas as concentrações iniciais de todos os elementos presentes em solução (nutrientes e $\mathrm{Cd}$ ) nos diferentes tratamentos, de modo a determinar a atividade iônica das principais formas dos elementos e avaliar o efeito do aumento da concentração de $\mathrm{P}$, na disponibilidade de $\mathrm{Cd}$.

Em ambos os experimentos, no final do período de exposição das plantas às doses de $\mathrm{Cd}$, realizouse a colheita, separando-se a parte aérea e raízes das plantas. No caso do experimento 1, retiraram-se amostras de raízes para avaliação da colonização micorrízica, conforme já descrito. O material foi colocado em estufa com circulação de ar a $65^{\circ} \mathrm{C}$ até massa constante para

R. Árvore, Viçosa-MG, v.31, n.5, p.783-792, 2007 
determinação da matéria seca, sendo posteriormente moído em moinho tipo Wiley equipado com peneira com malha de $0,38 \mathrm{~mm}$, para, então, sofrer digestão nítrico-perclórica e posterior análise da concentração de Cd nos tecidos por espectrofotometria de absorção atômica e de $\mathrm{P}$ por colorimetria, através do método fosfo-molibdato. As quantidades acumuladas de $\mathrm{Cd}$ na parte aérea e raízes das plantas foram calculadas com base nas concentrações do elemento e na produção de matéria seca da parte aérea e da raiz.

Os resultados foram submetidos à análise de variância, por meio do uso do programa estatístico SISVAR, e as equações de regressão foram determinadas pelo programa Table Curve 2D for Windows v. 5.01 (SYSTAT Software Inc). As doses críticas de toxidez de Cd em solução para redução em $50 \%$ da produção de matéria seca da parte aérea e raízes $\left(\mathrm{DCT}_{50}\right)$ foram estimadas com base nas equações de regressão obtidas em resposta às concentrações dos elementos em solução.

\section{RESULTADOS E DISCUSSÃO}

As plantas de trema expostas às soluções com excesso de Cd exibiram sintomas de fitotoxidez já aos 10 dias da exposição ao metal. Os sintomas progrediram com o tempo, de modo que, ao final dos 40 dias de exposição das plantas ao metal, foi observada a ocorrência de clorose internerval nas folhas mais jovens, semelhantemente à deficiência de Fe. Esses sintomas refletiram o crescimento das plantas, que mesmo nas doses mais baixas de Cd apresentaram crescimento reduzido (Figura 1ab). Esses sintomas foram menos acentuados nas plantas pré-tratadas com $\mathrm{P}$ do que naquelas com baixo Pe inoculação com $G$ etunicatum. A dose de $45 \mu \mathrm{mol} \mathrm{L}^{-1} \mathrm{Cd}$ apresentou elevada fitotoxicidade para a trema, causando queda das folhas e morte das plantas, independentemente de qualquer pré-tratamento (Figura 1a). Verificou-se também redução na massa radicular (Figura 1c) e escurecimento das raízes que se tornaram finas e quebradiças em concentrações superiores a $5 \mu \mathrm{mol} \mathrm{L}-1 \mathrm{Cd}$.
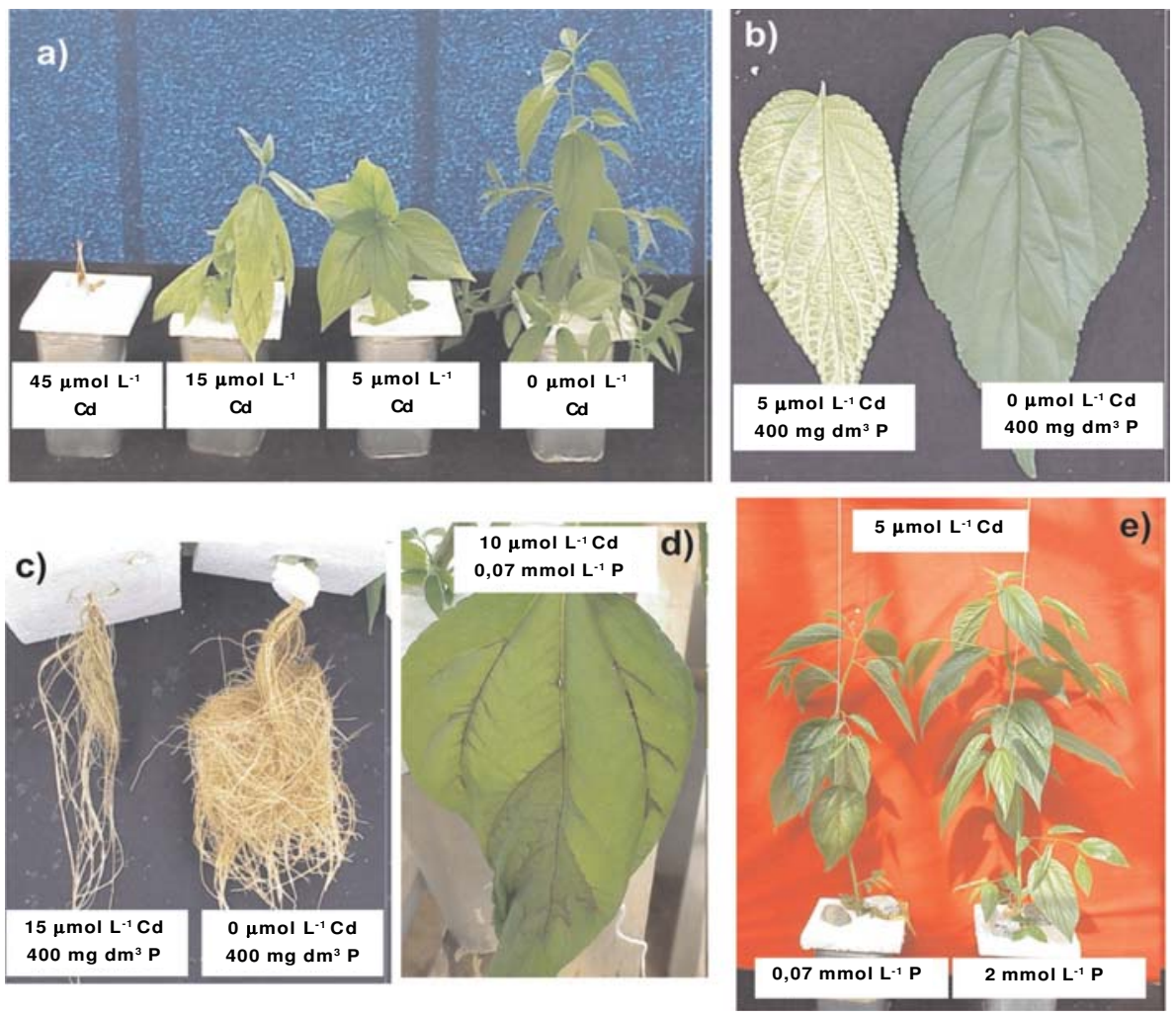

Figura 1 - Sintomas de toxidez de Cd em mudas de Trema micrantha cultivadas em solução nutritiva: a) efeito do aumento da concentração de $\mathrm{Cd}$; b) clorose foliar; c) redução no crescimento de raízes; d) pigmentos avermelhados próximos às nervuras; e e) efeito da concentração de $\mathrm{P}$ em solução.

Figure 1 - Cd toxicity symptoms in Trema micrantha seedlings grown in nutrient solution: a) effect on increasing Cd concentration; b) leaf chlorosis; c) root growth inhibition; d) internerval red pigmentation; e) effect of P concentration in solution.

R. Árvore, Viçosa-MG, v.31, n.5, p.783-792, 2007

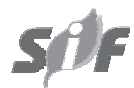


A elevação nas doses de Cd ocasionou acentuado decréscimo na produção de matéria seca das plantas, sendo esse efeito diferenciado para os pré-tratamentos de $\mathrm{P}$ e MA aplicados na formação das mudas (Figura 2a). Os pré-tratamentos com 200 e $400 \mathrm{mg} \mathrm{dm}^{-3} \mathrm{P}$ possibilitaram maior crescimento das plantas na dose mais baixa de $\mathrm{Cd}$ em solução $\left(5 \mu \mathrm{mol} \mathrm{L} \mathrm{L}^{-1}\right)$, em comparação com os demais pré-tratamentos. As doses críticas de toxidez em solução $\left(\mathrm{DCT}_{50}\right)$ para a matéria seca da parte aérea e raízes foram influenciadas pelos tratamentos. Na parte aérea, esta foi de $4,5 \mu \mathrm{mol} \mathrm{\textrm {L } ^ { - 1 }} \mathrm{Cd}$ em plantas sem $\mathrm{Pe}$ de $12 \mu \mathrm{mol} \mathrm{L}{ }^{-1} \mathrm{Cd}$ naquelas com $200 \mathrm{mg} \mathrm{dm}^{-3} \mathrm{P}$, indicando que o fornecimento prévio desse nutriente confere proteção ao excesso do $\mathrm{Cd}$ em solução. Tais valores de $\mathrm{DCT}_{50}$ são bastante inferiores aos valores encontrados na trema sob efeito tóxico de Zn (194 $\mu$ mol $\left.\mathrm{L}^{-1}\right)$, em ensaio realizado sob condições semelhantes (SOARES et al., 2006). As $\mathrm{DCT}_{50}$ de Cd nas plantas sem $\mathrm{P}$ e naquelas com $200 \mathrm{mg} \mathrm{dm}^{-3} \mathrm{P}$ correspondem a 0,51 e $1,35 \mathrm{mg} \mathrm{kg}^{-1}$, respectivamente, sendo estes inferiores aos valores comumente encontrados em solos contaminados por $\mathrm{Cd}\left(>3 \mathrm{mg} \mathrm{kg}^{-1}\right)$ (KABATA-PENDIAS e PENDIAS, 2001), evidenciando a elevada sensibilidade da trema ao Cd, o que inviabiliza o emprego dessa espécie na recomposição de áreas contendo concentrações elevadas desse elemento no solo.

A colonização micorrízica também foi severamente inibida pelo $\mathrm{Cd}$, reduzindo-se de $52 \%$ no controle para apenas $12 \%$ com $5 \mu \mathrm{mol} \mathrm{L}{ }^{-1} \mathrm{Cd}$ (Figura 2b). Portanto, esse metal tem elevado efeito tóxico para G. etunicatum em trema. A elevada sensibilidade desta simbiose ao Cd é relatada pela primeira vez, e isso tem dupla importância. Primeiramente, por indicar o grande potencial de dano ambiental que a contaminação com esse metal pode causar e, em segundo lugar, pela drástica redução na colonização micorrízica que limita a eventual contribuição da simbiose na proteção da planta hospedeira.
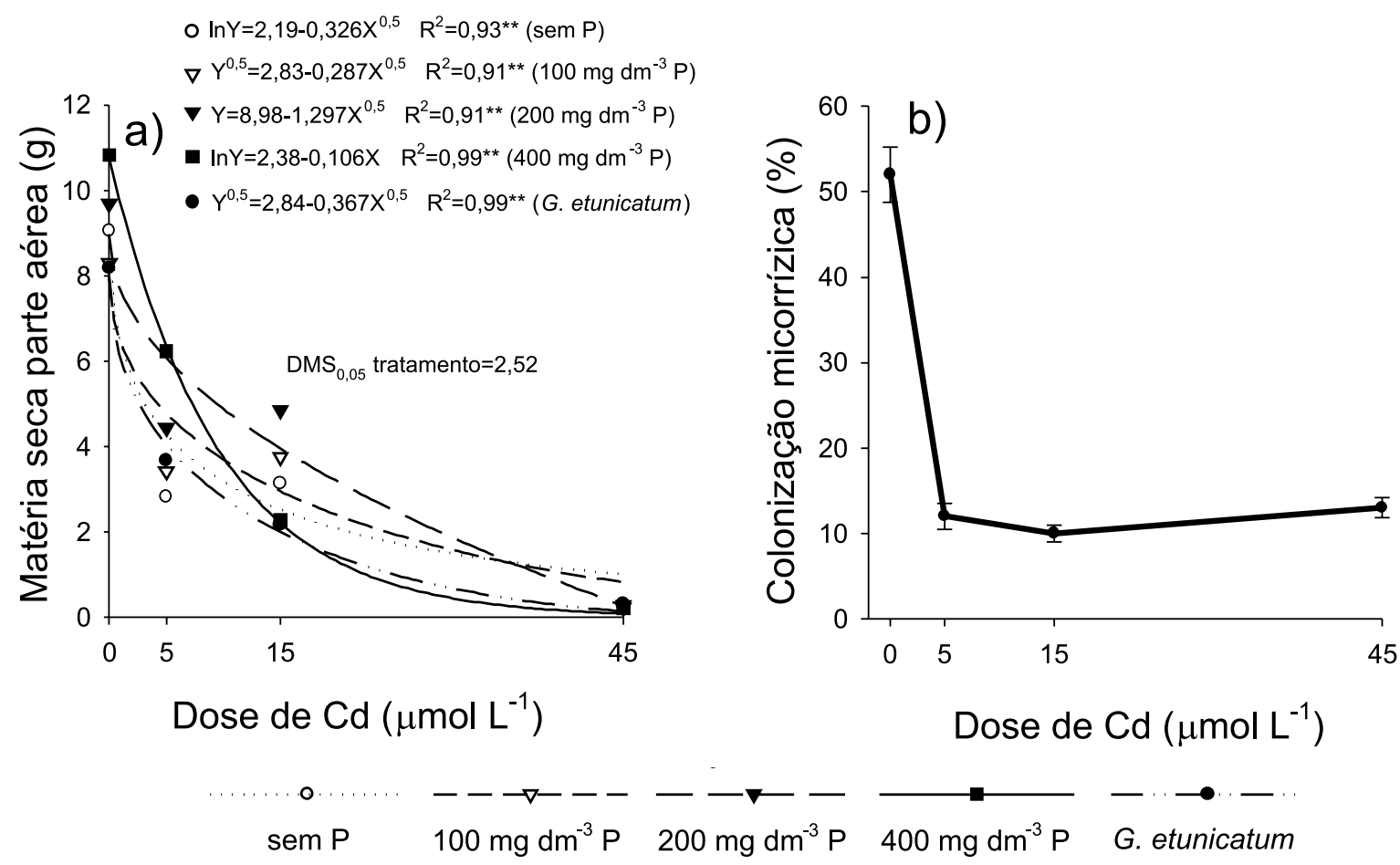

Figura 2 - Matéria seca da parte aérea (a) e colonização micorrízica (b) de mudas de Trema micrantha pré-colonizadas com G. etunicatum após 40 dias de crescimento em solução nutritiva contendo doses crescentes de Cd.

Figure 2 -Shoot dry matter (a) and mycorrhiza colonization (b) of Trema micrantha seedlings pre-colonized with G. etunicatum after 40 days of growth in nutrient solution with increasing Cd concentration. 
O aumento das doses de Cd resultou em elevação dos teores desse elemento nos tecidos (Figura 3a), atingindo-se valores estimados de até $1.228 \mathrm{mg} \mathrm{kg}^{-1} \mathrm{Cd}$ na matéria seca da parte aérea na dose de $26 \mu \mathrm{mol}$ $\mathrm{L}^{-1} \mathrm{Cd}$ (2,9 $\mathrm{mg} \mathrm{L}^{-1}$ de Cd em solução), evidenciando a elevada capacidade de absorção desse metal sem a função essencial conhecida às plantas. Os teores de Cd na trema são muito superiores aos valores de $380 \mathrm{mg} \mathrm{kg}^{-1}$ encontrados na parte aérea do eucalipto em $180 \mu \mathrm{mol} \mathrm{L}^{-1} \mathrm{Cd}$ em solução (SOARES et al., 2005) e na faixa de 5-30 $\mathrm{mg} \mathrm{kg}^{-1}$ considerada tóxica para a maioria das espécies (KABATA-PENDIAS e PENDIAS, 2001). Para visualizar melhor a relação entre o "status" nutricional de $\mathrm{P}$ das plantas e os teores de $\mathrm{Cd}$ na matéria seca da parte aérea, selecionaram-se os dados dos pré-tratamentos sem a adição de $\mathrm{P}$, inoculação com $G$. etunicatum e adição de $200 \mathrm{mg} \mathrm{dm}^{-3} \mathrm{P}$ na concentração de $15 \mu \mathrm{mol} \mathrm{L}^{-1}$ $\mathrm{Cd}$ (Figura 3b). Verificou-se que as plantas com baixo teor de $\mathrm{P}\left(2,1 \mathrm{~g} \mathrm{~kg}^{-1}\right)$ e colonizadas com $G$. etunicatum não diferiram quanto aos teores do metal na parte aérea (856 e $999 \mathrm{mg} \mathrm{kg}^{-1}$, respectivamente), que são 42 e $66 \%$ superiores aos teores encontrados nas plantas com alto $\mathrm{P}\left(4,1 \mathrm{~g} \mathrm{~kg}^{-1}\right)$, as quais continham "apenas" $601 \mathrm{mg} \mathrm{kg}^{-1}$ de Cd. Esses resultados indicam relação inversa entre o alto teor de $\mathrm{P}$ na planta e sua absorção de $\mathrm{Cd}$, podendo esse mecanismo nutricional estar envolvido na proteção da trema ao excesso desse metal como havia sido postulado por Wang et al. (2005). Na concentração de $45 \mu \mathrm{mol}$ $\mathrm{L}^{-1} \mathrm{Cd}$, também foram observadas diferenças entre os pré-tratamentos, mas esta foi letal para as plantas. A colonização com G. etunicatum não influenciou o teor de $\mathrm{P}$ nas condições deste estudo e também não afetou os teores de $\mathrm{Cd}$ na planta. Isso possivelmente decorre do elevado efeito fitotóxico deste metal sobre a colonização micorrízica.
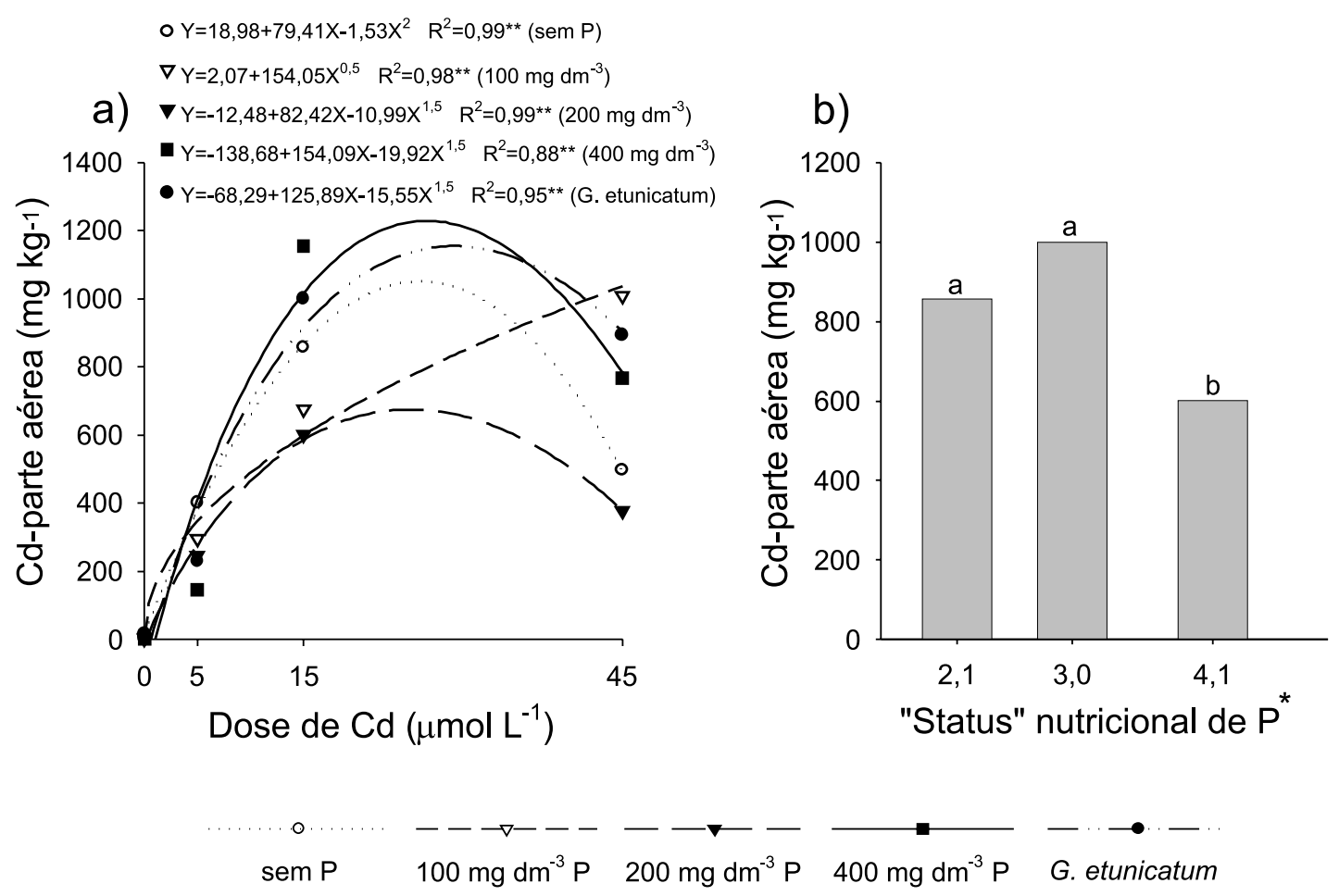

Figura 3 - a) Teores de Cd na parte aérea de mudas de Trema micrantha em resposta ao aumento de doses de Cd em solução, pré-tratamentos de P e inoculação com G. etunicatum; e b) efeito do "status" nutricional de $\mathrm{P}$ sobre os teores de Cd na parte aérea de T. micrantha em $15 \mu \mathrm{mol} \mathrm{L}{ }^{-1} \mathrm{Cd}$ (Scott-Knott 5\%). * Teores de P na planta em $\mathrm{g} \mathrm{kg}^{-1}$.

Figure 3 - a) Shoot Cd concentration in Trema micrantha in response to Cd in solution, $P$ pre-treatment and inoculation with G. etunicatum; b) effect of P nutritional status on Cd concentration in T. micrantha shoots at 15 umol $\mathrm{L}^{-1} \mathrm{Cd}$ (Scott-Knott 5\%). * Plant P concentration in $\mathrm{g} \mathrm{kg}^{-1}$.

R. Árvore, Viçosa-MG, v.31, n.5, p.783-792, 2007 
O aumento das doses de Cd também influenciou o acúmulo desse elemento na planta, com resposta quadrática $\left[\mathrm{y}=0,01+0,226 \mathrm{x}-0,0049 \mathrm{x}^{2}, \mathrm{R}^{2}=0,99 * * ; \mathrm{y}=\right.$ acúmulo de Cd $\left(\mathrm{mg}\right.$ planta $\left.\left.{ }^{-1}\right) ; \mathrm{x}=\operatorname{dose} \mathrm{Cd}\left(\mu \mathrm{mol} \mathrm{L}^{-1}\right)\right]$, porém sem efeito dos pré-tratamentos. Aquantidade de $\mathrm{Cd}$ acumulada aumentou de 0,01 (controle) para 2,3 $\mathrm{mg} \mathrm{planta}^{-1} \mathrm{com}$

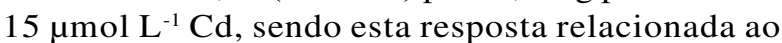
aumento dos teores desse elemento na planta. Na dose mais elevada de $\mathrm{Cd}\left(45 \mu \mathrm{mol} \mathrm{L}{ }^{-1}\right)$, acumulou-se apenas $0,25 \mathrm{mg}$ planta $^{-1}$, em razão da inibição na produção de matéria seca (Figura 2a). Apesar de não haver influência do "status" nutricional de $\mathrm{P}$ no acúmulo de $\mathrm{Cd}$ nos tecidos, pode-se verificar que houve efeito positivo da condição nutricional de $\mathrm{P}$ no crescimento da trema em baixas concentrações de $\mathrm{Cd}$ em solução, além de contribuir para a redução dos teores de Cd na planta.

No segundo experimento, observaram-se efeitos semelhantes para sintomas de fitotoxidez e crescimento das plantas devido ao Cd (Figura 1). Foram observados também a presença de pigmentos avermelhados próximos às nervuras das folhas (Figura 1d), o que, segundo
Sanità di Toppi e Gabbrielli (1999), está relacionado com o acúmulo de compostos fenólicos nos tecidos foliares em decorrência da toxicidade de Cd. Os sintomas de fitotoxidez foram mais evidentes em baixa concentração de $\mathrm{P}\left(0,07 \mathrm{mmol} \mathrm{L}^{-1}\right)$ e reduzidos em severidade quando se adicionou $\mathrm{P}$ na solução (Figura 1e).

$\mathrm{O}$ aumento do fornecimento de $\mathrm{P}$ estimulou a produção de matéria seca da parte aérea das plantas na ausência de Cd (Figura 4a). No entanto, em alta concentração de $\mathrm{Cd}$ essa resposta foi praticamente nula. Além disso, quando não se adicionou $\mathrm{P}$ na solução $\left(0,07 \mathrm{mmol} \mathrm{L}^{-1} \mathrm{P}\right)$, o crescimento da trema foi muito limitado e sem influência do $\mathrm{Cd}$. Em baixas concentrações de Cd em solução ( 5 e $10 \mu \mathrm{mol} \mathrm{L} \mathrm{L}^{-1}$ ), a adição de $\mathrm{P}$ favoreceu a produção de matéria seca (Figura 4a). Isso pode ser constatado por meio das doses críticas de toxidez de $\mathrm{Cd}\left(\mathrm{DCT}_{50}\right)$, em que o aumento da concentração de 0,5 para $2 \mathrm{mmol} \mathrm{L}^{-1}$ de $\mathrm{P}$ dobrou as $\mathrm{DCT}_{50}$, passando de 5,1 para $11,9 \mu \mathrm{mol} \mathrm{L}{ }^{-1} \mathrm{Cd}$. Esses resultados indicam que o maior fornecimento de $\mathrm{P}$ em solução contribui para amenizar a toxicidade do Cd para a trema.

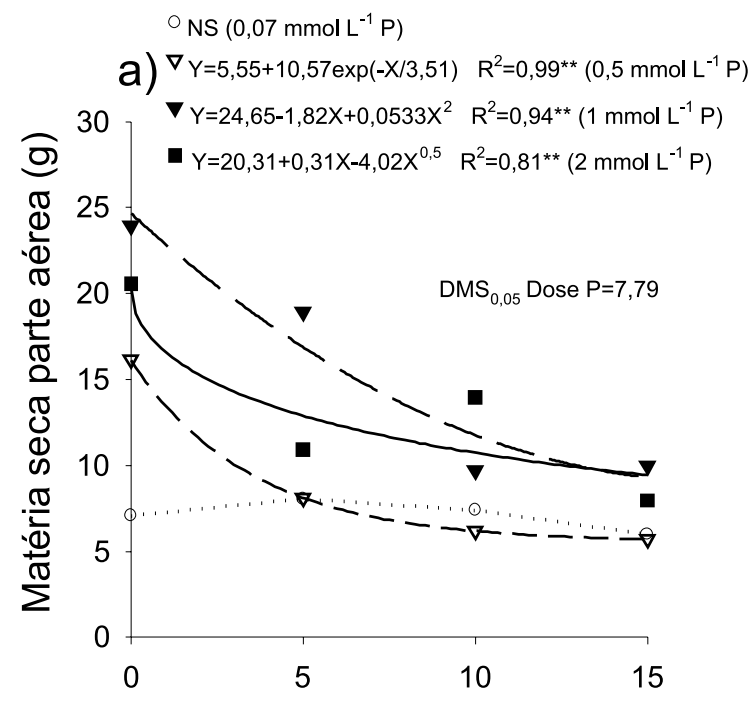

Dose de Cd $\left(\mu \mathrm{mol} \mathrm{L}{ }^{-1}\right)$

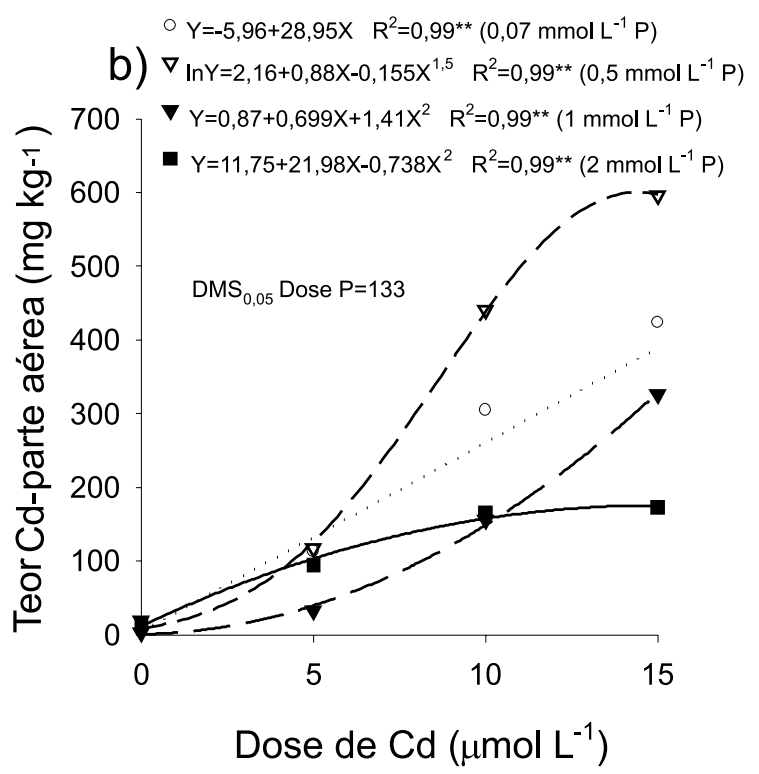

$\longrightarrow \mathrm{mmol} \mathrm{L} \mathrm{L}^{-1} \mathrm{P}$

Figura 4 - Matéria seca da parte aérea (a) e teores de Cd em mudas de Trema micrantha (b) em resposta ao aumento das doses de Cd e aplicação de $\mathrm{P}$ em solução nutritiva (** $\mathrm{p}=0,01 ; \mathrm{NS}=$ efeito não-significativo).

Figure 4 - Shoot dry matter ( $a$ ) and Cd concentration in Trema micrantha seedlings $(b)$ in response to increasing $C d$ and $P$ application in nutrient solution $(* * p=0,01 ; N S=$ non-significant effect $)$. 
A elevação da concentração de Cd promoveu incrementos nos teores desse elemento na planta, sendo esse efeito diferenciado nas doses de P (Figura 4b). Mesmo na dose mais elevada de $\mathrm{Cd}\left(15 \mu \mathrm{mol} \mathrm{L} \mathrm{L}^{-1}\right)$, a adição de $2 \mathrm{mmol} \mathrm{L}^{-1} \mathrm{P}$ reduziu em mais de $70 \%$ os teores desse elemento na parte aérea, em relação à dose de $0,5 \mathrm{mmol} \mathrm{L}^{-1} \mathrm{P}$, indicando a influência da nutrição fosfática na redução dos teores foliares de Cd nessa planta. Não há indicações de mecanismos envolvidos nessa resposta, mas uma possibilidade é que o aumento de $\mathrm{P}$ possa contribuir para a formação de compostos de Cd menos móveis na planta (BROWN et al., 1995), resultando em baixa translocação do $\mathrm{Cd}$ absorvido pelas raízes, para a parte aérea da planta. Na maior concentração de Cd em solução, a quantidade total de metal acumulada reduziu de 6,64 para 4,99 $\mathrm{mg}$ planta $^{-1}$, ao se elevar o fornecimento de 0,5 para $2 \mathrm{mmol} \mathrm{L}^{-1}$ de P. Além disso, a razão do acúmulo de $\mathrm{Cd}$ na parte aérea e raízes (PA/ $\mathrm{R})$ reduziu de 0,90 nas plantas com baixo $P$ para apenas 0,47 no tratamento com alto $P$. Esses resultados indicam que o aumento no fornecimento de $\mathrm{P}$ reduz a quantidade acumulada de Cd na planta e decresce a distribuição relativa desse elemento na parte aérea, podendo, dessa forma, estar contribuindo para a menor toxicidade do Cd para a trema.
Uma das possibilidades para se explicar o efeito do fornecimento de P na amenização da toxicidade de Cd seria na redução da disponibilidade do metal para as plantas (KABATA-PENDIAS e PENDIAS, 2001). No entanto, a especiação iônica da solução indicou que a elevação da concentração de $\mathrm{P}$ de 0,07 para $2 \mathrm{mmol} \mathrm{L}^{-1}$ não interferiu nas principais formas de $\mathrm{Cd}$ consideradas disponíveis para a planta (Tabela 1). A maior parte do Cd encontrase complexada comEDTA $\left(\mathrm{CdEDTA}^{2-}\right)$, portanto com elevada biodisponibilidade, conforme demonstrado por Greman et al. (2003). Ao contrário disso, formas de Cd consideradas menos disponíveis para a absorção como o $\mathrm{CdHPO}_{4}$ (ALLOWAY, 1993) apresentam baixa atividade em solução $\left(<0,1 \mu \mathrm{mol} \mathrm{L}{ }^{-1}\right)$. Esses resultados indicam que o aumento de $\mathrm{P}$ na solução não interfere, de modo relevante, na biodisponibilidade de $\mathrm{Cd}$, sendo a ação amenizante desse nutriente resultado de efeitos fisiológicos ou nutricionais na planta e não devido à redução da absorção de $\mathrm{Cd}$. Cálculos da razão $\mathrm{P} / \mathrm{Cd}$ na matéria seca da parte aérea, tanto no segundo quanto no primeiro experimento, mostraram que esta reduz drasticamente de 260 (média do controle) para apenas 7,5 com $5 \mu \mathrm{mol} \mathrm{L}{ }^{-1} \mathrm{Cd}$. Em nenhum caso, a adição de $P$ resultou em balanço mais favorável da relação P/Cd na planta, não sendo, dessa forma, um mecanismo atenuador da toxicidade do $\mathrm{Cd}$ para a trema.

Tabela 1 - Especiação de Cd em solução nutritiva de Clark sob concentrações crescentes de P, empregando-se o software Visual Minteq com $\mathrm{PCO}_{2}=0,03$ atm e $\mathrm{pH}=5,5$

Table 1 - Cd speciation in Clark nutrient solution at increasing P concentration by the Visual Minteq program at $\mathrm{PCO}_{2}$ $=0.03$ atm and $\mathrm{pH}=5.5$

\begin{tabular}{|c|c|c|c|c|}
\hline \multirow{2}{*}{$\begin{array}{l}\text { Doses Cd solução } \\
0 \mu \mathrm{mol} \mathrm{L}{ }^{-1} \mathrm{Cd}\end{array}$} & \multicolumn{4}{|c|}{ Especiação de Cd (\%)† } \\
\hline & $\mathrm{Cd}^{2+}$ & CdHEDTA $^{-}$ & CdEDTA $^{2-}$ & Outras formas (incluindo $\mathrm{CdHPO}_{4}$ ) \\
\hline$+0,07 \mathrm{mmol} \mathrm{L}^{-1} \mathrm{P}$ & - & - & - & - \\
\hline$+0,5 \mathrm{mmol} \mathrm{L}^{-1} \mathrm{P}$ & - & - & - & - \\
\hline$+1,0 \mathrm{mmol} \mathrm{L} \mathrm{L}^{-1} \mathrm{P}$ & - & - & - & - \\
\hline$+2,0 \mathrm{mmol} \mathrm{L}^{-1} \mathrm{P}$ & - & - & - & - \\
\hline \multicolumn{5}{|l|}{$5 \mu \mathrm{mol} \mathrm{L}{ }^{-1} \mathrm{Cd}$} \\
\hline$+0,07 \mathrm{mmol} \mathrm{L} \mathrm{L}^{-1} \mathrm{P}$ & 0,1 & 0,5 & 99 & $<0,1$ \\
\hline$+0,5 \mathrm{mmol} \mathrm{L}^{-1} \mathrm{P}$ & 0,1 & 0,5 & 99 & $<0,1$ \\
\hline$+1,0 \mathrm{mmol} \mathrm{L}^{-1} \mathrm{P}$ & 0,1 & 0,5 & 99 & $<0,1$ \\
\hline$+2,0 \mathrm{mmol} \mathrm{L}^{-1} \mathrm{P}$ & 0,1 & 0,5 & 99 & $<0,1$ \\
\hline \multicolumn{5}{|l|}{$10 \mu \mathrm{mol} \mathrm{L}-1 \mathrm{Cd}$} \\
\hline$+0,07 \mathrm{mmol} \mathrm{L}-1 \mathrm{P}$ & 0,1 & 0,5 & 99 & $<0,1$ \\
\hline$+0,5 \mathrm{mmol} \mathrm{L}^{-1} \mathrm{P}$ & 0,1 & 0,5 & 99 & $<0,1$ \\
\hline$+1,0 \mathrm{mmol} \mathrm{L}^{-1} \mathrm{P}$ & 0,1 & 0,5 & 99 & $<0,1$ \\
\hline$+2,0 \mathrm{mmol} \mathrm{L}^{-1} \mathrm{P}$ & 0,1 & 0,5 & 99 & $<0,1$ \\
\hline \multicolumn{5}{|l|}{$15 \mu \mathrm{mol} \mathrm{L}{ }^{-1} \mathrm{Cd}$} \\
\hline$+0,07 \mathrm{mmol} \mathrm{L} \mathrm{L}^{-1} \mathrm{P}$ & 0,2 & 0,5 & 99 & $<0,1$ \\
\hline$+0,5 \mathrm{mmol} \mathrm{L} \mathrm{L}^{-1} \mathrm{P}$ & 0,2 & 0,5 & 99 & $<0,1$ \\
\hline$+1,0 \mathrm{mmol} \mathrm{L}^{-1} \mathrm{P}$ & 0,2 & 0,5 & 99 & $<0,1$ \\
\hline$+2,0 \mathrm{mmol} \mathrm{L}^{-1} \mathrm{P}$ & 0,1 & 0,5 & 99 & $<0,1$ \\
\hline
\end{tabular}

$\dagger$ Porcentagem da concentração total do componente.

R. Árvore, Viçosa-MG, v.31, n.5, p.783-792, 2007 


\section{CONCLUSÕES}

OCd teve elevada toxicidade para a Trema micrantha e para a simbiose desta com Glomus etunicatum.

A melhoria da nutrição fosfatada da T. micrantha contribuiu para a amenização da toxidez de $\mathrm{Cd}$ sobre essa planta, enquanto a colonização das mudas com G. etunicatum não teve efeito protetor para elas nas condições estudadas.

O aumento no fornecimento de $\mathrm{P}$ reduziu a absorção e a quantidade acumulada de $\mathrm{Cd}$ na parte aérea das plantas, conferindo proteção contra a toxicidade de Cd para a T. micrantha.

\section{REFERÊNCIAS}

ALLOWAY, B. J. Heavy metals in soils. New York: John Wiley, 1993. 339p.

BARCELÓ, J.; POSCHENRIEDER, Ch. Respuestas de las plantas a la contaminación por metales pesados. Suelo y Planta, v.2, p.345-361, 1992.

BROWN, S. L. et al. Zinc and cadmium uptake by hyperaccumulator Thlaspi caerulescens grown in nutrient solution. Soil Science Society of America Journal, v.59, n.1, p.125-133, 1995.

CAMPOS, M. L. et al. Determinação de cádmio, cobre, cromo, níquel, chumbo e zinco em fosfatos de rocha. Pesquisa Agropecuária Brasileira, v.40, n.4, p.361-367, 2005.

CHRISTIE, P.; LI, X.; CHEN, B. Arbuscular mycorrhiza can depress translocation of zinc to shoots of host plants in soils moderately polluted with zinc. Plant and Soil, v.261, n.2/3, p.209-217, 2004.

CLARK, R. B. Characterization of phosphates in intact maize roots. Journal of Agriculture and Food Chemistry, v.23, n.3, p.458-460, 1975.

COBBETT, C. Heavy metals and plants - model systems and hyperaccumulators. New Phytologist, v.159, p.289-293, 2003.

CUI, Y. et al. Exposure to metal mixtures and human health impacts in a contaminated area in Nanning, China. Environment

International, v.31, p.784-790, 2005.
GAUR, A.; ADHOLEYA, A. Prospects of arbuscular mycorrhizal fungi in phytoremediation of heavy metal contaminated soils. Current Science, v.86, n.4, p.528-534, 2004.

GERDEMANN, J. B.; NICOLSON, T. H. Spores of mycorrhizal Endogone species extracted from soil by wet sieving and decanting.

Transactions British Mycological Society, v.46, p.235-246, 1963.

GIOVANNETTI, M.; MOSSE, B. An evaluation of techniques to measure vesicular-arbuscular infection in roots. The New Phytologist, v.84, n.3, p.489-500, 1980.

GREMAN, H. et al. Ethylenediaminedissuccinate as a new chelate for environmentaly safe enhanced lead phytoextraction. Journal of Environmental Quality, v.32, n.4, p.500-506, 2003.

JONER, E. J.; LEYVAL, C. Uptake of ${ }^{109}$ Cd by roots and hyphae of a Glomus mosseae/Trifolium subterraneum mycorrhiza from soil amended with high and low concentrations of cadmium. New Phytologist, v.135, n.2, p.353-360, 1997.

KABATA-PENDIAS, A.; PENDIAS, H. Trace elements in soils and plants. Boca Raton: CRC Press, 2001. 413p.

KRUPA, P.; KOZDRÓJ, J. Accumulation of heavy metals by ectomycorrhizal fungi colonizing birch trees growing in an industrial desert soil. World Journal of Microbiology \&

Biotechnology, v.20, n.4, p.427-430, 2004.

KOSKE, R. E.; GEMMA, J. N. A modified procedure for staining roots to detect VA mycorrhizas. Mycological Research, v.92, n.4, p.486-488, 1989.

MARQUES, T. C. L. L. S. M.; MOREIRA, F. M. S.; SIQUEIRA, J. O. Crescimento e absorção de metais em mudas de espécies arbóreas em solo contaminado com metais pesados. Pesquisa Agropecuária Brasileira, v.35, n.1, p.121-132, 2000.

MEHARG, A. A. The mechanistic basis of interactions between mycorrhizal associations and toxic metal cátions. Mycological Research, v.107, n.11, p.1253-1265, 2003.

R. Árvore, Viçosa-MG, v.31, n.5, p.783-792, 2007 
PAIVA, H. N.; CARVALHO, J. G.; SIQUEIRA, J. O. Efeito de $\mathrm{Cd}, \mathrm{Ni}, \mathrm{Pb}$ e $\mathrm{Zn}$ sobre mudas de cedro (Cedrela fissilis Vell.) e de ipê-roxo (Tabebuia impetiginosa (Mart.) Standley) em solução nutritiva. Revista Árvore, v.24, n.4, p.369-378, 2000.

PARON, M. E.; SIQUEIRA, J. O.; CURI, N. Fungos micorrízicos, fósforo e nitrogênio no crescimento inicial da trema e do fededoso.

Revista Brasileira de Ciência Solo, v.21, n.4, p.567-574, 1997.

RIBEIRO FILHO, M. R. et al. Metais pesados em solos de rejeitos de indústria de processamento de zinco. Revista Brasileira de Ciência do Solo, v.23, n.3, p.453-464, 1999.

SANITÀ di TOPPI, L.; GABBRIELLI, R. Response to cadmium in higher plants.

Environmental Experimental Botany, v.41, n.2, p.105-130, 1999.
SOARES, C.R.F.S. et al. Fitotoxidez de cádmio para Eucalyptus maculata e E. urophylla em solução nutritiva. Revista Árvore, v.29, n.2, p.175-183, 2005.

SOARES, C. R. F. S. et al. Micorriza arbuscular e nutrição fosfática na toxidez de zinco para a trema [Trema micrantha (L.) Blum.]. Revista Brasileira de Ciência do Solo, v.30, n.4, p.665-675, 2006.

WANG, F.; LIN, X.; YIN, R. Heavy metal uptake by arbuscular mycorrhizas of Elsholtzia splendens and the potential for phytoremediation of contaminated soil. Plant and Soil, v.269, p.225-232, 2005.

YANG, X. et al. Influx, transport, and accumulation of cadmium in plant species grown at different $\mathrm{Cd}^{2+}$ activities. Journal Environmental Science Health, v.30, n.4, p.569-583, 1995. 